COMPRENDRE

\section{L'imagerie numérique rapide par caméra haute vitesse}

Julien ROMANN - Photon Lines, Saint-Grégoire, France- ju-romann@photonlines.com

L'imagerie numérique rapide est un domaine riche en innovations tournées vers l'accroissement constant de la cadence d'acquisition, de la sensibilité, ou encore de la flexibilité des dispositifs permettant l'observation jusqu'alors impossible de phénomènes extrêmement brefs. Parmi ces dispositifs, les caméras « haute vitesse » regroupent une part majeure de ce marché dynamique en couvrant à elles seules une grande variété d'applications.

\footnotetext{
L

es technologies permettant l'observation de phénomènes rapides par acquisition d'images à haute vitesse se sont développées sur près d'un siècle pour donner lieu à une large gamme de solutions. Aujourd'hui dominées par les caméras numériques « haute vitesse », ces solutions d'imagerie rapide répondent à des problématiques applicatives variées, qu'elles soient propres au domaine militaire, médical, industriel, sportif, ou à ceux de la biologie ou de la physique fondamentale. Une telle étendue d'applications n'empêche pourtant pas le marché de l'imagerie numérique rapide de poursuivre sa croissance (estimée à 2,2 \% entre 2016 et 2022 pour le marché global des caméras rapides [1]), cela dans une constante nécessité de développer des systèmes plus performants et de répondre à des problématiques nouvelles.

Bien que présentant toutes des limites intrinsèques induisant des compromis en termes de vitesse, de résolution et de durée d'acquisition, les différentes technologies d'imagerie rapide ont toujours su évoluer de manière à repousser ces limites et minimiser de tels compromis. En effet,
}

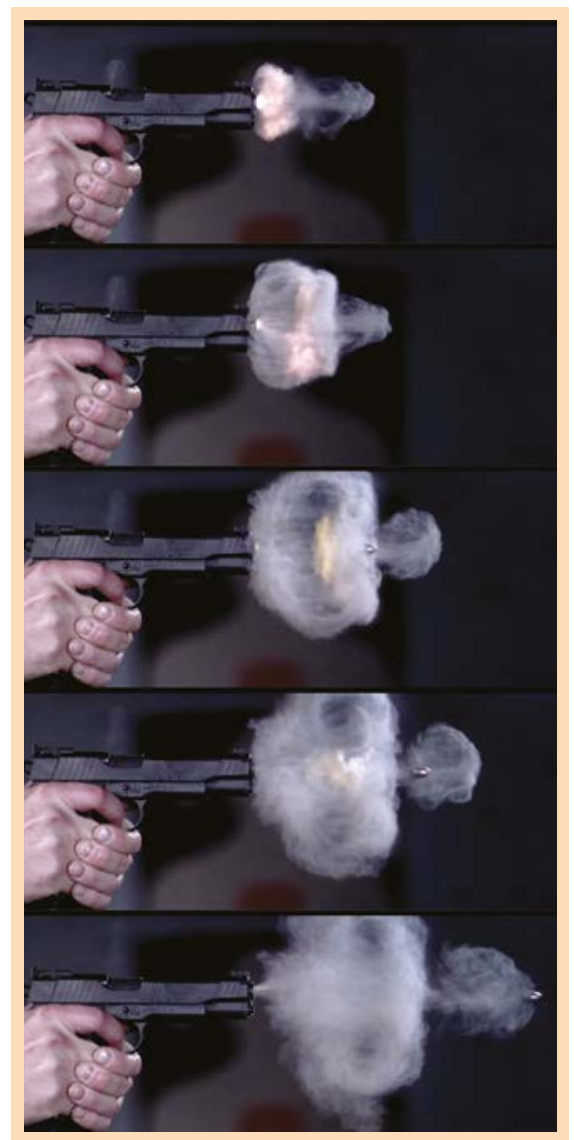

Figure 1. Coup de feu filmé à haute vitesse (VRI Phantom V2010, cadence de 73000 fps). Discovery, https://www.youtube.com/ watch?v=7y9apnbI6GA.

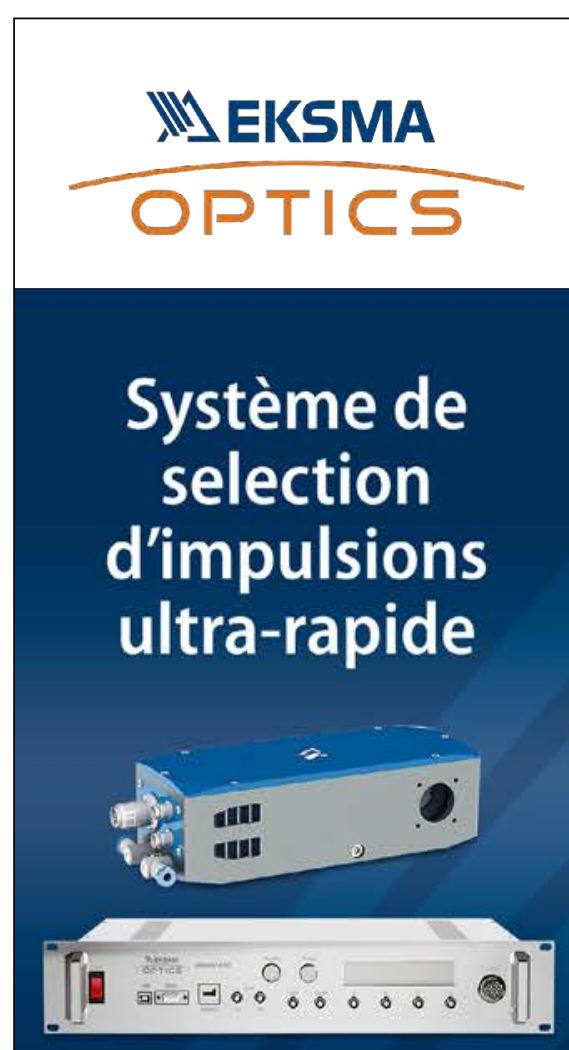

Sélectionner

des trains d'impulsions

supérieurs au $\mathrm{MHz}$

\section{Cellule de Pockels double cristal de BBO}

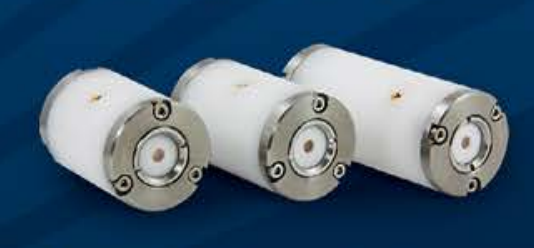

Design fiable pour les lasers industriels de forte puissance

www.eksmaoptics.com

Représenté en France par:

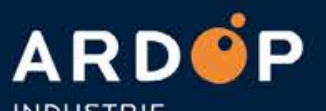

INDUSTRIE

+33169632609 | www.ardop.com 
des premières caméras à tubes cathodiques aux révolutions successives induites par les capteurs CCD ( 1970) et CMOS $(\sim 1990)$ et jusqu'au récent développement des capteurs d'image à stockage in situ (ISIS), cette évolution technologique s'est globalement traduite de façon parallèle par une course à la vitesse et par une plus grande flexibilité des systèmes.

Cet article a pour objectif de présenter les fondements ainsi que les spécificités propres aux principales technologies de l'imagerie numérique rapide (caractérisée par des cadences d'acquisition allant de la centaine à plusieurs millions d'images par seconde) par caméra haute vitesse.

\section{Fondements, capteurs et performances}

Dans les grandes lignes, le fonctionnement d'une caméra numérique haute vitesse n'est pas différent de celui d'une caméra numérique classique. La scène observée est d'abord focalisée par un objectif caractérisé par une longueur focale et une ouverture relative. Le champ d'observation, la longueur focale de l'objectif, la distance de travail et la dimension du capteur sont

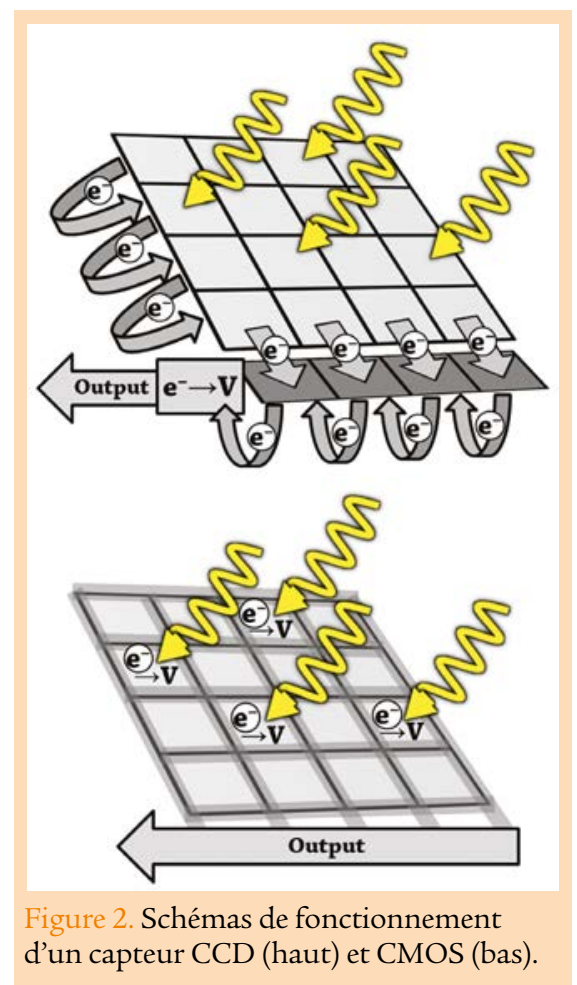

alors des grandeurs liées par la même relation. La focalisation de la scène par l'objectif est effectuée sur un capteur numérique appartenant en général soit à la famille technologique des capteurs CCD (charged coupled device), soit à celle des capteurs CMOS (complementary metal oxide semiconductor), familles dont les sous-technologies déterminent la méthode employée pour convertir les photons incidents en signal numérique. La vitesse maximale d'une caméra numérique, exprimée en nombre d'images par seconde (ou frames per second, fps), et sa résolution (nombre de pixels exposés) sont interdépendantes. Une telle caméra pourra ainsi fonctionner à pleine résolution jusqu'à ce que la cadence maximale qui lui est propre soit atteinte.

Les capteurs CCD ont permis dans les années 1980 d'éliminer certains artefacts propres aux caméras analogiques et ont longtemps dominé le marché des capteurs optiques. Un capteur CCD est constitué d'une matrice de capacités MOS au sein de laquelle chaque pixel génère un puits de potentiel permettant de stocker les photoélectrons issus du rayonnement incident. Ces photoélectrons sont ensuite transférés de pixel à pixel de façon séquentielle sous l'effet de la tension de grille. Le temps nécessaire à l'exécution de ce transfert et des conversions charge-signal puis analogique-numérique réalisées en sortie de capteur constitue une limite notable à l'intérêt de la technologie CCD dans le cadre de l'imagerie haute vitesse. Bien que bénéficiant d'une haute sensibilité, les capteurs CCD permettent généralement des cadences maximales de quelques centaines au millier de fps et restent relativement coûteux.

Souvent considérés comme la solution optimale en imagerie haute vitesse, les capteurs CMOS bénéficient d'une architecture leur conférant davantage de flexibilité que les capteurs CCD vis-à-vis du perpétuel compromis cadence - résolution. En effet, la conversion charge-signal réalisée en simultané sur l'ensemble des pixels de la matrice CMOS, permet à ces capteurs d'atteindre des cadences significativement plus élevées (plusieurs centaines à plusieurs dizaines de milliers de fps à pleine résolution), et ce à des coûts inférieurs. La possibilité de réduire la zone du capteur exploitée en définissant une région d'intérêt (ROI) permet d'augmenter encore la cadence d'acquisition, et ce jusqu'à un million de fps (1 Mfps) à résolution minimale pour certaines caméras CMOS.

Une autre différence de la technologie CMOS par rapport à la technologie CCD concerne le mécanisme d'obturation (shutter). Alors que les capteurs CCD fonctionnent en global shutter où tous les pixels sont exposés à la lumière au même instant, les capteurs CMOS proposent deux types de shutter. Le rollingshutter implique une exposition par balayage « ligne par ligne » des pixels à la lumière. Ce type de shutter maximise la sensibilité, mais induit à haute cadence une distorsion des images d'objets en mouvement en raison du décalage temporel lié à l'exposition ligne après ligne. Le global shutter est l'alternative permettant de s'affranchir de ce phénomène, mais le nombre accru de transistors nécessaires au niveau de chaque pixel diminue la sensibilité ainsi que la résolution spatiale. Des progrès ont néanmoins été réalisés ces dernières années afin de réduire ces diminutions de sensibilité et de résolution, si bien que la majorité des capteurs CMOS haute vitesse sont aujourd'hui équipés d'un global shutter.

\section{Cadence, résolution, dynamique, mémoire... et bande passante}

Comme nous l'avons vu, le capteur d'une caméra rapide impose intrinsèquement un compromis entre cadence et résolution. Mais l'architecture d'un capteur n'est pas le seul paramètre induisant un tel compromis. En effet, l'acquisition à haute cadence d'informations provenant d'un grand nombre de pixels se traduit par la nécessité de diriger et de stocker un flux de données élevé. La capacité de stockage d'une caméra rapide est importante car elle ajoute à la cadence et à la résolution d'autres paramètres 


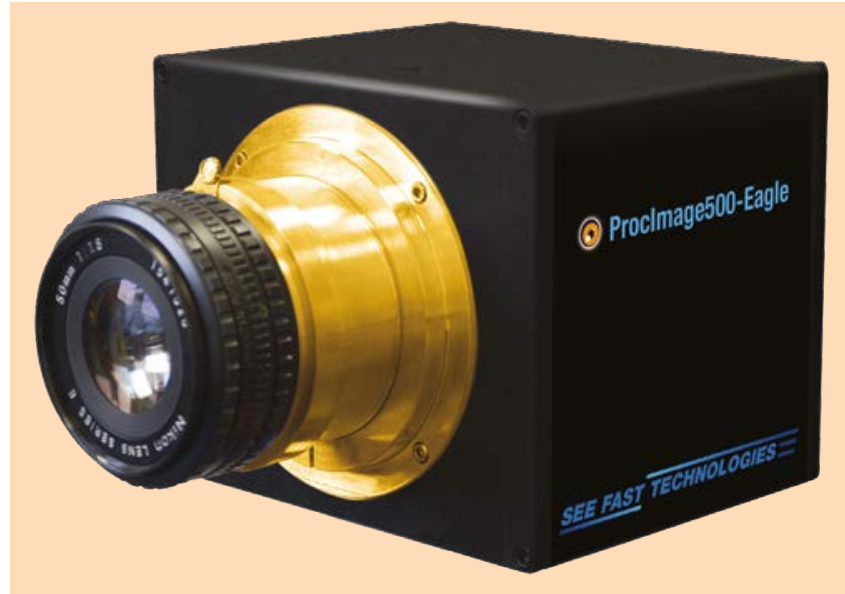

limitants tels que la dynamique (en bit) ou encore la durée d'acquisition déterminant tous deux le volume de données à stocker. De ce fait, une même relation définit l'interdépendance de ces paramètres. Ainsi, pour une caméra numérique rapide comportant un capteur de $N$ pixels, d'une dynamique de $d$ bits, filmant à une cadence de $V$ fps durant un temps de $T$ secondes, la mémoire minimum (M octets ou « bytes ») nécessaire pour stocker les données générées peut être déterminée comme suit :

$$
M=\frac{1}{8}(N \times d \times V \times T)
$$

Pour une caméra numérique rapide donnée, la pleine résolution de son capteur, sa dynamique et la mémoire de stockage dont elle dispose sont des paramètres fixes. Le choix auquel l'utilisateur est confronté se traduit donc par un compromis nécessaire entre cadence, définition ou non d'une ROI, dimension de l'éventuelle ROI et durée d'acquisition, compromis répondant aux contraintes inhérentes au capteur et à la mémoire de la caméra.

Enfin, un dernier paramètre peut également limiter le flux de données généré par une acquisition rapide d'images. Ce paramètre intervient en particulier dans le cas des caméras ne disposant pas de mémoire interne et dont le flux de données doit être transféré en «temps réel » (à la cadence d'acquisition) vers un périphérique de stockage externe. Dès lors, certaines configurations matérielles induisent une limitation du flux de données par la bande passante (exprimée en gigabit par seconde, noté Gbps) caractérisant l'interface

\begin{tabular}{|l|r|r|}
\hline INTERFACE & BANDE PASSANTE & CÂBLE \\
\hline USB 2.0 & 0,48 Gbps & $3 \mathrm{~m}$ \\
\hline GigE Vision & 1 Gbps & $100 \mathrm{~m}$ \\
\hline Camera Link & 2 Gbps & $10 \mathrm{~m}$ \\
\hline Camera Link HS & $3,13 \mathrm{Gbps}$ & $15 \mathrm{~m}$ \\
\hline USB 3.0 & 5 Gbps & $3 \mathrm{~m}$ \\
CoaXpress & 6,25 Gbps & $130 \mathrm{~m}$ \\
\hline GigE Vision 2.0 & 10 Gbps & $100 \mathrm{~m}$ \\
\hline
\end{tabular}

Tableau 1. Bande passante théorique et longueur de câble maximale d'interfaces « vision » courantes.

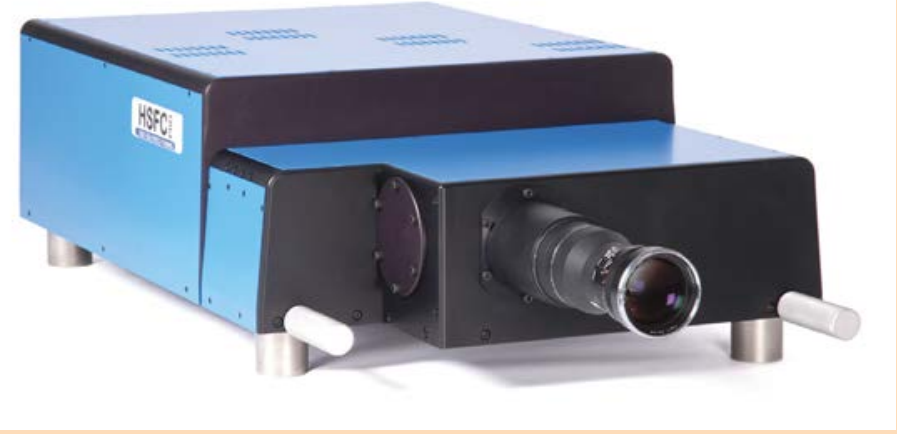

Figure 3. Caméra ProcImage 500 Eagle.

$\triangle$ Figure 4. Caméra ultra-rapide intensifiée (iCCD) pco HSFC pro. entre la caméra rapide et le périphérique de stockage externe. Ces caméras rapides ne disposant pas de mémoire interne sont souvent conçues pour des applications de vision industrielle, leurs cadences maximales allant généralement de plusieurs centaines au millier de fps. La bande passante de ces caméras dépend donc du type d'interface de communication dont elles sont équipées. Le tableau 1 détaille la bande passante théorique ainsi que la longueur de câble

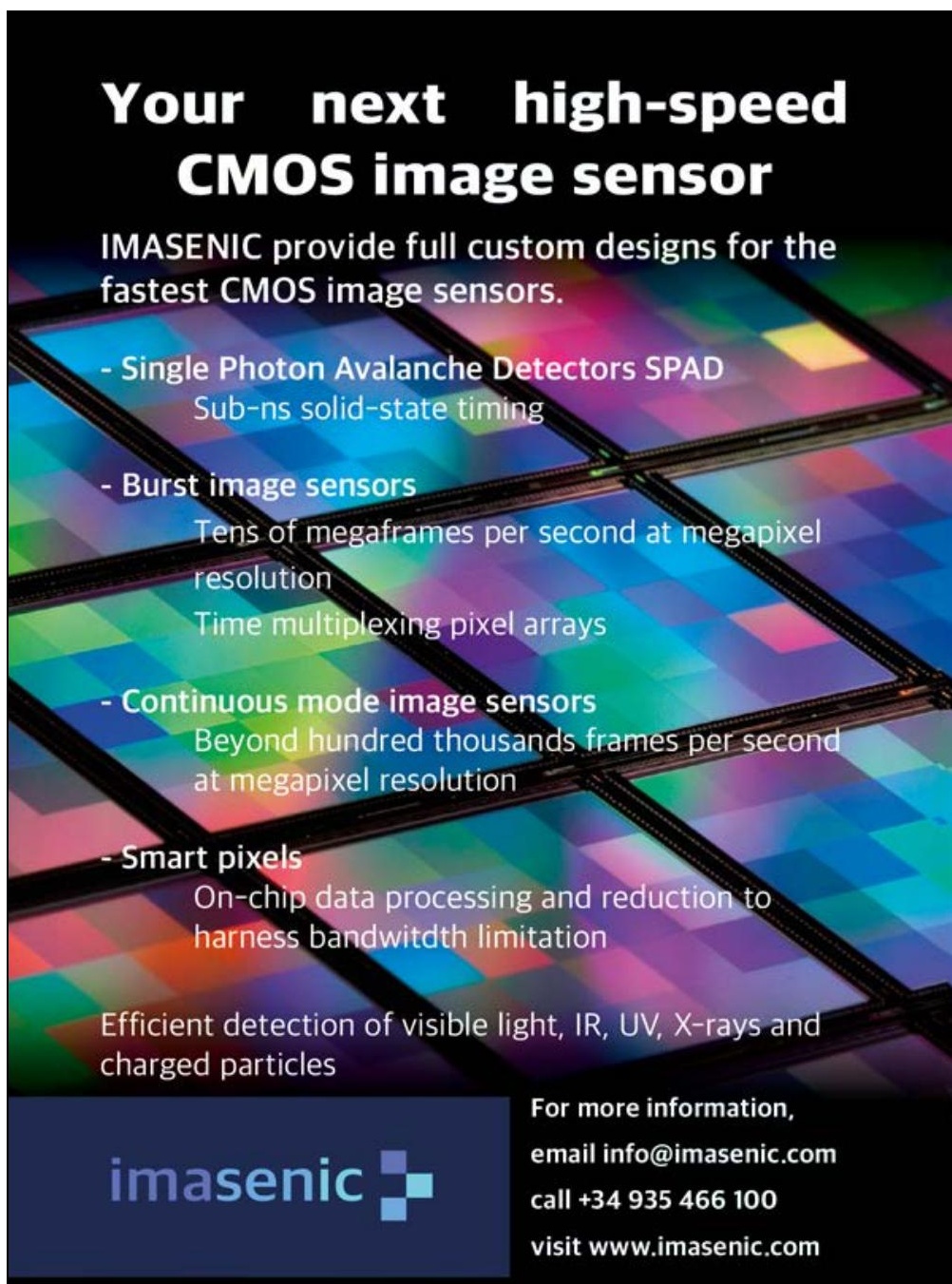




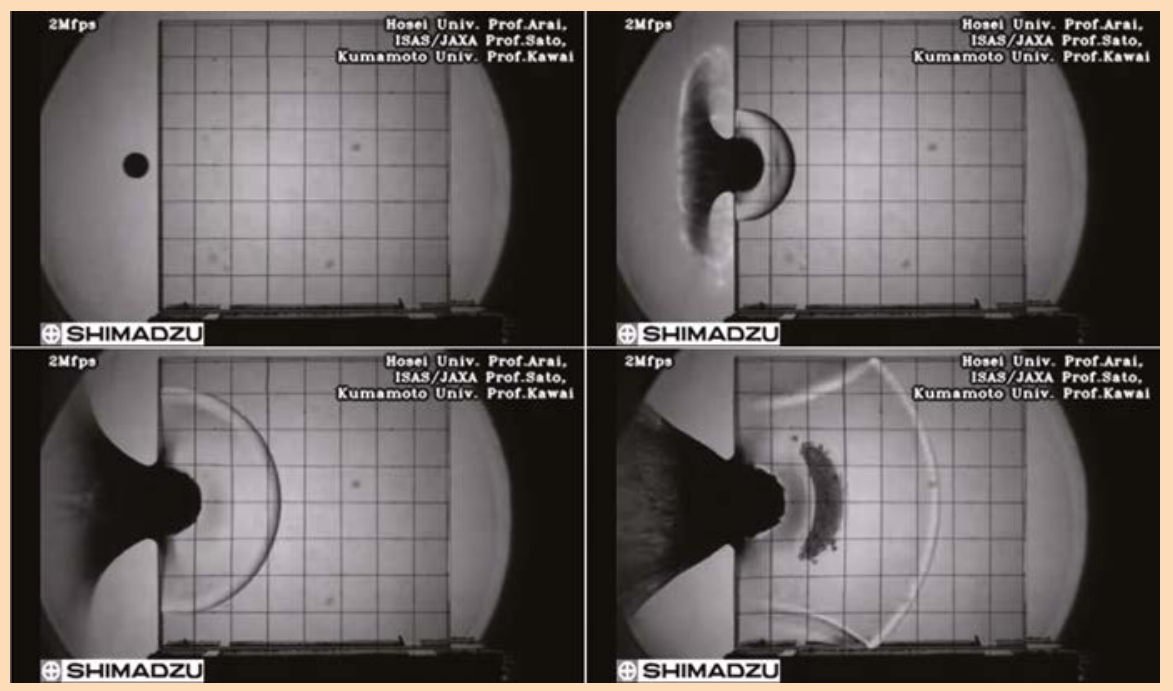

Prise de vues ultra-haute vitesse (2 Mfps) de la collision d'un laminé de polycarbonate avec une sphère de nylon (Shimadzu, Hosei Univ. Prof. Arai, ISAS/JAXA Prof. Satho, Kumamoto Univ. Prof. Kawai).

maximum caractérisant les principales interfaces présentes dans les caméras rapides industrielles [2].

Il est enfin intéressant de noter que certaines caméras rapides ont la capacité de réaliser des traitements d'images en « temps réel » (à la cadence d'acquisition de la caméra), et ce afin de réduire le volume de données à transmettre en ne conservant que les informations que l'utilisateur juge pertinentes (présence, comptage, dimensions, forme, coordonnées, déplacement d'objets d'intérêt, etc...). Utilisant des processeurs logiques de type FPGA (field-programmable gate array), les caméras See Fast Technologies ProcImage 250 / 500 Eagle sont une bonne illustration de cette stratégie qui contourne donc en partie la limitation imposée par la bande passante.

\section{Repousser les limites}

Différentes stratégies ont été exploitées afin de repousser les limites propres à l'imagerie rapide « classique » et ainsi atteindre des vitesses d'acquisition toujours plus élevées. Parmi elles, certaines approches impliquent l'utilisation d'intensificateurs. En effet, une cadence extrêmement rapide implique un temps d'obturation très court et donc un déficit d'intensité intégrée au niveau pour le système XXRapidFrame), limitant ainsi drastiquement la durée de chaque acquisition.

Une autre approche intéressante est celle du capteur d'images à stockage in situ (ISIS). Au sein d'un capteur CCD ou CMOS classique, la cadence d'acquisition est en grande partie limitée par le temps de transfert du signal vers les composants situés hors du capteur. Le principe du capteur ISIS est d'intégrer les éléments de stockage du signal au sein même du capteur (in situ) et à proximité directe de chaque pixel, réduisant ainsi considérablement le temps de transfert pour chaque image. Ainsi, les caméras Shimadzu HPV-2 et HPV-X2 comportent des capteurs ISIS respectivement de type CCD permettant une cadence maximale de $1 \mathrm{Mfps}$ à pleine résolution $(312 \times 260$ pixels $)$ pour un maximum de 100 images par acquisition, et de type CMOS permettant une cadence maximale de $10 \mathrm{Mfps}$ à pleine résolution $(400 \times 250$ pixels $)$ pour un maximum de 256 images par acquisition [3].

\section{Conclusion}

Les innovations repoussant toujours plus loin les limites de flexibilité ou de vitesse en imagerie numérique rapide se poursuivent à un rythme soutenu. Même le récent développement de la photographie à détection compressée (CUP), dont la cadence d'acquisition extrême de $100000 \mathrm{Mfps}$ [4] permet l'observation du déplacement d'un front lumineux, ouvre encore de vastes perspectives technologiques. Une question méritera donc peutêtre bientôt d'être posée : reste-t-il quelque chose de trop rapide pour être observé ? 